\title{
Complex networks emerging during choir singing
}

\author{
Viktor Müller,${ }^{1}$ Julia A.M. Delius, ${ }^{1}$ and Ulman Lindenberger ${ }^{1,2,3}$ \\ ${ }^{1}$ Center for Lifespan Psychology, Max Planck Institute for Human Development, Berlin, Germany. ${ }^{2}$ European University \\ Institute, San Domenico di Fiesole (FI), Italy. ${ }^{3}$ Max Planck UCL Centre for Computational Psychiatry and Ageing Research, \\ London, England, and Berlin, Germany
}

Address for correspondence: Dr. Viktor Müller, Center for Lifespan Psychology, Max Planck Institute for Human Development, Lentzeallee 94, 14195 Berlin, Germany. vmueller@mpib-berlin.mpg.de

Choir singing is positively associated with well-being and quality of life, and requires the coordination of physiological systems within and across individuals. Informed by models of interpersonal action coordination, we delineate the network topography of choir singing by analyzing cross-frequency couplings and within-frequency couplings (WFCs) of respiratory, cardiac, vocalizing, and motor subsystems. We find that respiratory and cardiac subsystems synchronize with one another during singing and are coupled to oscillatory vocalizing patterns, and to the handmovement oscillations of the choir's conductor. The choir's cross-frequency connections are particularly strong when singing a canon in parts, apparently supporting the interaction and coordination of the different canon entries. In contrast, WFCs are more pronounced when singing the same canon in unison. We conclude that the temporal coordination dynamics of the observed subsystems form part of the functional substrate for choir singing. During singing, the choir functions as a superordinate system, or superorganism, that imposes boundary conditions on the dynamic features of the individual singers.

Keywords: phase synchronization; cross-frequency coupling; heart rate variability; graph-theoretic approach; social networks

\section{Introduction}

Choral singing is a highly coordinated form of social behavior that has been found to promote social and mental well-being, ${ }^{1-4}$ and to enhance cooperation in groups. ${ }^{5-7}$ Singing in a choir requires synchronization of several components or subsystems such as respiration, heart rate variability (HRV), and vocalization, both within and across individuals. Furthermore, the conductor's hand movements play an important role during choir singing in controlling the onset and coordination of the different singers, who often sing in different voices, such as soprano, alto, tenor, and bass. In a previous analysis restricted to within-frequency couplings (WFCs), we showed how structural properties of the music, such as singing in unison versus singing in different entries, are reflected in the choir's network topography. ${ }^{8}$ However, many of the couplings that define the choir as a system are likely to operate across frequencies. Hence, to obtain a more com- plete picture of the functional choir network, crossfrequency couplings (CFCs) need to be considered as well. Quantitative methods to examine CFC have been available for some time $e^{9-13}$ and are increasingly applied in the neurosciences. ${ }^{14-17}$

We contend that the choir as a superordinate system imposes boundary conditions on its constituents, that is, on the different voices and the individual singers who make up these voices. In biological systems, the kinetics of lower level constituents are often underidentified unless higher level components provide boundary and initial conditions. Hence, to understand the structure and function of such systems, both bottom-up and topdown directions of causation need to be taken into account. ${ }^{18}$ In the case of the choir, the network dynamics of each individual singer are likely to be influenced by the function of his or her voice for the choir as a whole. Hence, we hypothesized that the choir effectively functions as a superordinate 
system, or superorganism, ${ }^{18}$ that imposes boundary conditions on each individual singer.

To investigate choir singing, we need to represent the network topography of its coupling dynamics in a comprehensive fashion. So far, few studies have investigated synchronization of autonomic responses between individuals carrying out joint activities. Konvalinka et al. found synchronized arousal, as assessed by heart rate dynamics, between active participants and related spectators in a Spanish firewalking ritual. ${ }^{19}$ Interestingly, participants did not synchronize their arousal with other members of the audience. In a study using a paced breathing protocol, Censi et al. ${ }^{20}$ found that the synchronization patterns between respiration and cardiovascular variability are fleeting, and affected by breathing rhythms. In a study with resting human subjects, Tzeng et al..$^{21}$ observed cardio-respiratory couplings at both low (0.04$0.15 \mathrm{~Hz})$ and high $(0.15-0.40 \mathrm{~Hz})$ frequency ranges. As known, these frequency ranges are related to the sympathetic and parasympathetic branches of the autonomic nervous system (ANS). There is also evidence that low-frequency oscillations of respiratory and/or cardiac systems may reflect both sympathetic and parasympathetic activity, while high-frequency oscillations primarily represent parasympathetic autonomic influences. ${ }^{22}$ Little, if anything, is known about the directionality or causality of this coupling or about interactions between frequencies. Schäfer and colleagues showed cardiorespiratory synchronization of several locking ratios, with respiration driving HRV. ${ }^{23}$ Grape et $a .^{2}$ reported that professional singers showed an increase in the low- and high-frequency spectral power of HRV during singing, whereas amateur singers did not. In another study, ${ }^{24}$ it was found that HRV and respiration mirrors music (singing) structure and there was a clear tendency toward an entrainment between singers in terms of heart rate (HR) acceleration and deceleration when singing a simple structure in unison. Our own previous analysis of choir singing ${ }^{8}$ was the first to investigate synchronization patterns of cardiac and respiratory activity between singing individuals. In this work, we show that synchronization in respiration and HRV between the choir members was significantly higher during singing than in the resting state, indicating that these synchronization patterns were specific to singing.
The present investigation uses the same data set, but refines and extends our previous analyses in three major ways. First, in addition to respiratory and cardiac signals, we also included the singers' vocalizations and the conductor's hand movements in our analyses. Second, we extended our analyses from WFC to CFC, which opens up a wide range of hitherto unobservable coupling relations. Third, the four subsystems were not analyzed as separate entities but simultaneously, allowing us to observe all interaction patterns across subsystems and frequencies. WFC and CFC were analyzed conjointly, using a newly developed network construction method. ${ }^{12,25}$ The approach integrates all subsystems and frequency components into a common network, thereby rendering the network topography of a highly synchronized social activity with unprecedented richness.

In summary, the present study examines the oscillatory network topography of choir singing as indexed by cardiac, respiratory, vocalizing, and gestural activity. We investigated two questions, one general and the other specific. At the general level, we examined whether the respiratory and cardiac subsystems of the singers would synchronize during singing and couple to the vocalizing patterns of the singers, and to the hand movements of the choir's conductor. At the specific level, we predicted that the oscillatory networks within individual singers would vary as a function of choir context, in line with the hypothesis that the choir functions as a superordinate system. To test these hypotheses, we focused our analyses on differences in the network topographies of the choir, and of each individual singer, as a function of whether a canon was sung in unison or in parts (i.e., with different entries).

\section{Methods}

\section{Participants}

As described in our earlier paper, ${ }^{8}$ we recruited five men and seven women (age range $=23.06-56.68$ years; $M=35.7 ; \mathrm{SD}=10.69$ ) from the choir of the Max Planck Institute for Human Development in Berlin, Germany. The group consisted of a conductor and 11 singers. The choir was founded 2 years prior to the study and consisted of a mixed group of approximately 12-15 staff members, researchers, and students from different departments who had joined the choir in the course of its existence. Most choir members did not know each other well before 
joining the choir and only interacted closely during the weekly 2-h rehearsals. The study was approved by the ethics committee of Max Planck Institute for Human Development (Berlin), and performed in accordance with the ethical standards laid down in the 1964 Declaration of Helsinki. All subjects volunteered for this experiment and gave their written informed consent prior to their inclusion in the study.

\section{Procedure}

As described previously, ${ }^{8}$ the participants were aligned in a predetermined position with the 11 singers facing the conductor and standing in two rows. The testing session began and ended with a relaxation condition. In this study, we focused on the data acquired during singing of the canon "Signor Abbate" in B major (by Ludwig van Beethoven) in three experimental variations: (1) singing the canon in unison $\left(\mathrm{C}_{\mathrm{un}}\right),(2)$ singing the canon with three individual parts at regular intervals with eyes open $\left(\mathrm{C}_{\mathrm{eo}}\right)$, and (3) singing the canon with three individual parts at regular intervals with eyes closed $\left(\mathrm{C}_{\mathrm{ec}}\right.$, the conductor sang along in the third part). All recordings were obtained with the subjects in a standing position and lasted $5 \mathrm{~min}$ per condition. The tasks were separated by 3 -min breaks. As evident from our previous study, ${ }^{8}$ this period between the experimental conditions is sufficient for stabilization of the measurements. An order effect of conditions was not expected. The choir members were instructed to avoid physical contact and verbal communication during singing. Nonverbal communication (e.g., eye contact) was not forbidden.

\section{Data acquisition and analysis}

The electrocardiogram (ECG), respiratory movement, and vocal audio signals were recorded simultaneously from all participants during each of the three conditions. In addition, the conductor's hand movements were measured throughout (see Ref. 8 for details).

The QRS complexes in the ECG signals were used to identify beat locations. Once the timing of beats was determined, an instantaneous HR signal was created. Vocal and conductor's hand movement signals were adjusted to the low-frequency respiration and HRV time series using the time-frequency analytic wavelet transform with energy normalization and consecutive averaging of the spectral power within the frequency range of the maximal ampli- tude of frequency response (see Fig. 1 for details). Thereafter, all signals were down-sampled to $4 \mathrm{~Hz}$. The Spencer's 15-Point Moving Average method was used to smooth a time series in order to highlight the underlying structure. In the signals, the power spectral density was calculated using fast Fourier transform with a Hamming window to determine spectral peaks. In all, 10 different frequency components were chosen for further analyses based on the spectral peaks and with regard to the fixed relation between the frequencies (1:2, 1:3, 2:3, etc.): 0.025 , $0.050,0.075,0.100,0.125,0.150,0.200,0.250,0.300$, and $0.400 \mathrm{~Hz}$.

Our analyses were conducted in a data-driven manner. To investigate phase synchronization within and between the frequencies, we applied an analytic complex-valued Morlet wavelet transform to compute the instantaneous phase in the frequency range from 0 to $2 \mathrm{~Hz}$ in steps of $0.0025 \mathrm{~Hz}$. WFC and CFC were determined using the ICI algorithm described in our previous study. ${ }^{8}$ This was extended to the calculation of CFC in terms of a generalized phase difference: $\Delta \Phi(t)=n \phi_{m}(t)-m \phi_{n}(t)$, where $m$ and $n$ are integers, and $\phi_{m, n}$ are phases of two oscillators. In the case of WFC with $\phi_{m}=$ $\phi_{n}$, the phase difference $\Delta \Phi$ is calculated in the same way by setting $m=n=1$. ICI is an asymmetric or directed coupling measure indicating both the common (absolute) and the positive or leading influence exerted by phase synchronization and ranges between 0 and 1 .

\section{Network construction and network properties}

For network construction, we used WFC and CFC between all choir members determined for the four subsystems (HRV, respiration, voice, and conductor's hand movements) oscillating at 10 different frequencies each time. Thus, each choir member evinced 30 nodes ( 3 systems $\times 10$ frequencies), whereas the conductor yielded 20 additional nodes due to the signals from the hand movement sensors. Thus, the common network contained 380 nodes altogether, covering all possible interactions between the choir members and including interactions within and between the subsystems and oscillation frequencies. In order to determine the network properties, we set the cost level (ratio of the number of actual connections divided by the maximum possible number of connections in the network) to $25 \%$, which makes it possible to 
A
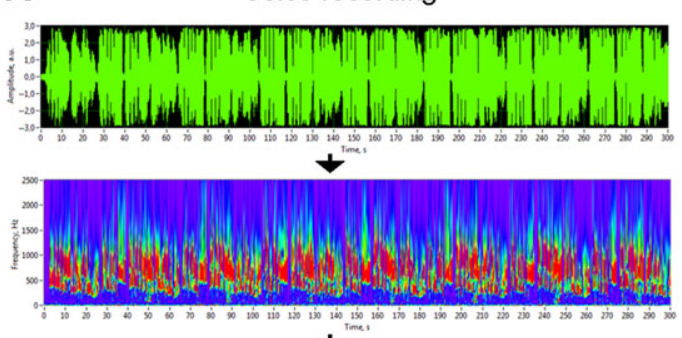

$+$

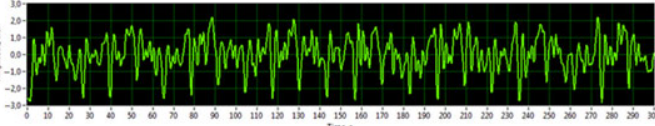

C

Respiration
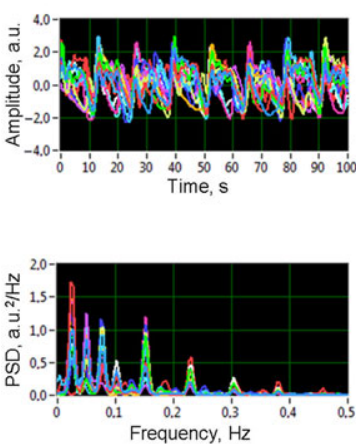

HRV
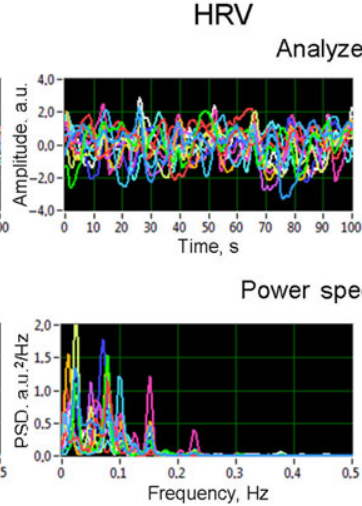

B

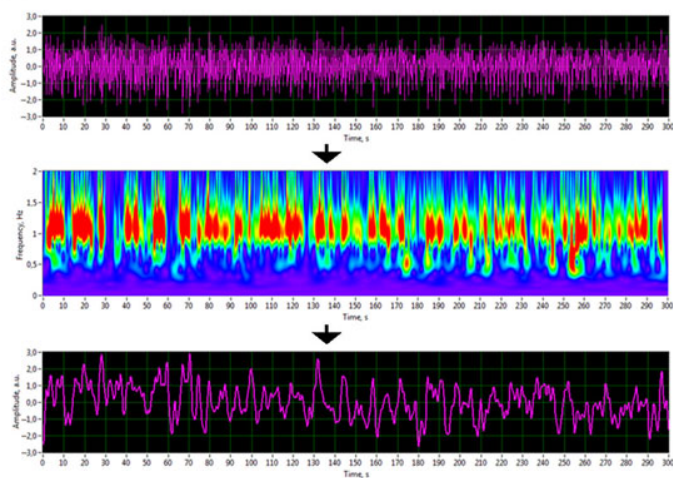

Hand movements

Voice recording

Hand movements
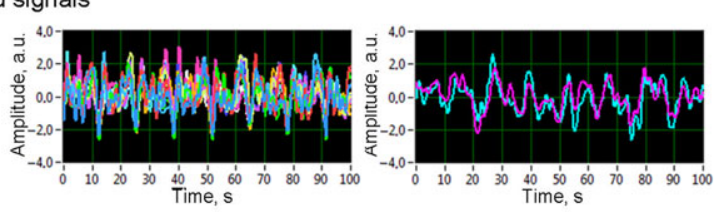

Power spectral density
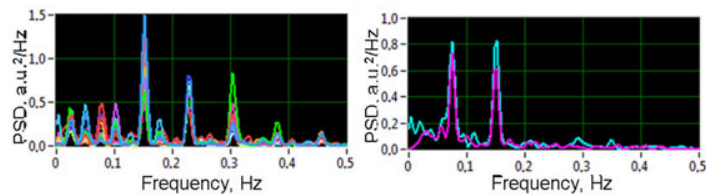

Figure 1. Transformation of voice and hand movement signals and power spectral density of analyzed signals. (A) Raw signal of a voice recording (top), wavelet transform of the voice signal (middle), and new voice signal derived from the wavelet-transformed signal by averaging the wavelet power across the frequency bins (bottom). (B) Equivalently processed signals of the movements of one of the conductor's hands. (C) Analyzed signals representing different subsystems investigated in the study (respiration, heart rate variability, voice production, and conductor's hand movements) and corresponding power spectral densities. The participants are indicated by different colors. HRV, heart rate variability.

investigate sparse networks. The connectivity threshold was always higher than the significance level determined by the surrogate data procedure (i.e., networks at this cost or sparsity level always included significant connections and had relatively similar number of edges). This allowed a more accurate examination of the network topology in the different canon conditions. Surrogate data were created in two ways: (1) by random permutations of the original time series and (2) by phase permutation of the time series. The resulting threshold of $I C I=0.23$, corresponding to the cost level of $25 \%$, was always higher than the determined significance level in both surrogate data procedures.

WFC and CFC strengths. As ICI is a directed weighted measure, we obtained the nodes' in- and out-strengths, in which the in-strength is the sum of weights of all incoming connections $\left(w_{j i}\right), k_{i}^{\text {in }}=$ $\sum_{j \in N} w_{j i}$ and the out-strength is the sum of weights of all outgoing connections $\left(w_{i j}\right), k_{i}^{\text {out }}=\sum_{j \in N} w_{i j}$ For statistical analyses, we used the out-strengths only, which were calculated for WFC and CFC connections separately, and also grouped and summed across different nodes or links referring to the system, frequency, and choir members (also within and between them). To be able to compare within- and between-member strength, the latter was averaged across the individuals.

\section{Modularity analyses and $Z-P$ parameter space.}

Community structures for directed networks as well as indices of modularity $(M)$, within-module degree $\left(Z_{i}\right)$, and participation coefficients $\left(P_{i}\right)$ were 
determined using the Brain Connectivity Toolbox. ${ }^{26}$ The optimal community structure is a subdivision of the network into nonoverlapping groups of nodes in a way that maximizes the number of within-module edges, and minimizes the number of between-module edges. $M$ is a statistic that quantifies the degree to which the network may be subdivided into such clearly delineated groups or modules. It is given for directed networks by the formula: ${ }^{27}$

$$
M^{\rightarrow}=\frac{1}{l} \sum_{i, j \in N}\left\lfloor a_{i j}-\frac{k_{i}^{\text {in }} k_{i}^{\text {out }}}{l}\right\rfloor \cdot \delta_{m_{i} m_{j}},
$$

where $l=\sum_{i j} a_{i j}$ is the number of edges in the graph, and $a_{i j}$ is defined to be one if there is an edge from $j$ to $i$, and zero otherwise, $k_{i}^{\text {in }}$ and $k_{i}^{\text {out }}$ are the in-and out-degrees of the node $i$, and $\delta_{m_{i} m_{j}}$ is the Kronecker delta, which is equal to one if $m_{i}=m_{j}$, and zero otherwise. High modularity values indicate strong separation of the nodes into modules. $M=0$ if nodes are placed into modules at random or if all nodes are in the same cluster. ${ }^{27}$ To test the modularity of the empirically observed networks, we compared them to the modularity distribution $(N=100)$ of random networks, that is, to simulated networks with the same number of nodes and edges as the original network. ${ }^{28}$

The within-module degree $Z_{i}$ indicates how well node $i$ is connected to other nodes within the module $m_{i}$. As shown in Ref. 29, it is determined by:

$$
Z_{i}=\frac{k_{i}\left(m_{i}\right)-\bar{k}\left(m_{i}\right)}{\sigma^{k\left(m_{i}\right)}}
$$

where $k_{i}\left(m_{i}\right)$ is the within-module degree of node $i$ (the number of links between $i$ and all other nodes in $\left.m_{i}\right)$, and $\bar{k}\left(m_{i}\right)$ and $\sigma^{k\left(m_{i}\right)}$ are the mean and standard deviation of the within-module degree distribution of $m_{i}$.

The participation coefficient $P_{i}$ describes how well the nodal connections are distributed across different modules: ${ }^{29}$

$$
P_{i}=1-\sum_{m \in M}\left(\frac{k_{i}\left(m_{i}\right)}{k_{i}}\right)^{2},
$$

where $M$ is the set of modules, $k_{i}\left(m_{i}\right)$ is the number of links between node $i$ and all other nodes in module $m_{i}$, and $k_{i}$ is the total degree of node $i$ in the network. Correspondingly, $P_{i}$ of a node $i$ is close to one if its links are uniformly distributed among all the modules, and zero if all of its links lie within its own module. $Z_{i}$ and $P_{i}$ values form a so-called $Z-P$ parameter space and are characteristic for the different roles of the nodes in the network. ${ }^{29}$ These roles in the $Z-P$ parameter space could be defined as follows: nonhub provincial nodes (low $Z_{i}$ and $P_{i}$ values), nonhub connector nodes (low $Z_{i}$ and high $P_{i}$ values), hub provincial nodes (high $Z_{i}$ and low $P_{i}$ values), and hub connector nodes (high $Z_{i}$ and $P_{i}$ values). In this context, hubs are responsible for intramodular connectivity and contain multiple connections within a module; while connector nodes maintain intermodular connectivity and are responsible for links between the modules.

\section{Statistical analysis}

To compare the three canon conditions and the within- and between-member connectivity of the three subsystems, we averaged the between-member connectivity strengths for each individual. Together with the within-member connectivity strength, we subjected them to a four-way repeated-measures ANOVA with the four within-subject factors: member (within-member versus between-member connection strengths), condition $\left(\mathrm{C}_{\mathrm{un}}, \mathrm{C}_{\mathrm{ec}}\right.$, and $\left.\mathrm{C}_{\mathrm{eo}}\right)$, system (respiration, HRV, and voice), and frequency (10 frequencies). We performed this ANOVA separately for the WFC and CFC strengths. The hand movement data were not included in the statistical analyses because they were only available for the conductor. In the common network, the $Z_{i}$ and $P_{i}$ values determined by the modularity analysis were subjected to a three-way repeated-measures ANOVA with the three within-subject factorscondition, system, and frequency. When necessary, Greenhouse-Geisser epsilons were used in all ANOVAs for nonsphericity correction. The Scheffé test was employed for the post-hoc testing of condition differences. All statistical analyses were carried out using IBM SPSS Statistics 23.0 (SPSS Inc., Chicago, IL).

\section{Results}

Based on both WFC and CFC, we constructed a common WFC-CFC network that combines coupling among all the signals, that is, all singers' respiration, HRV, voices, and the conductor's hand movements, oscillating at 10 different frequencies and representing different autonomic systems of choir participants in relation to each other and to 
A
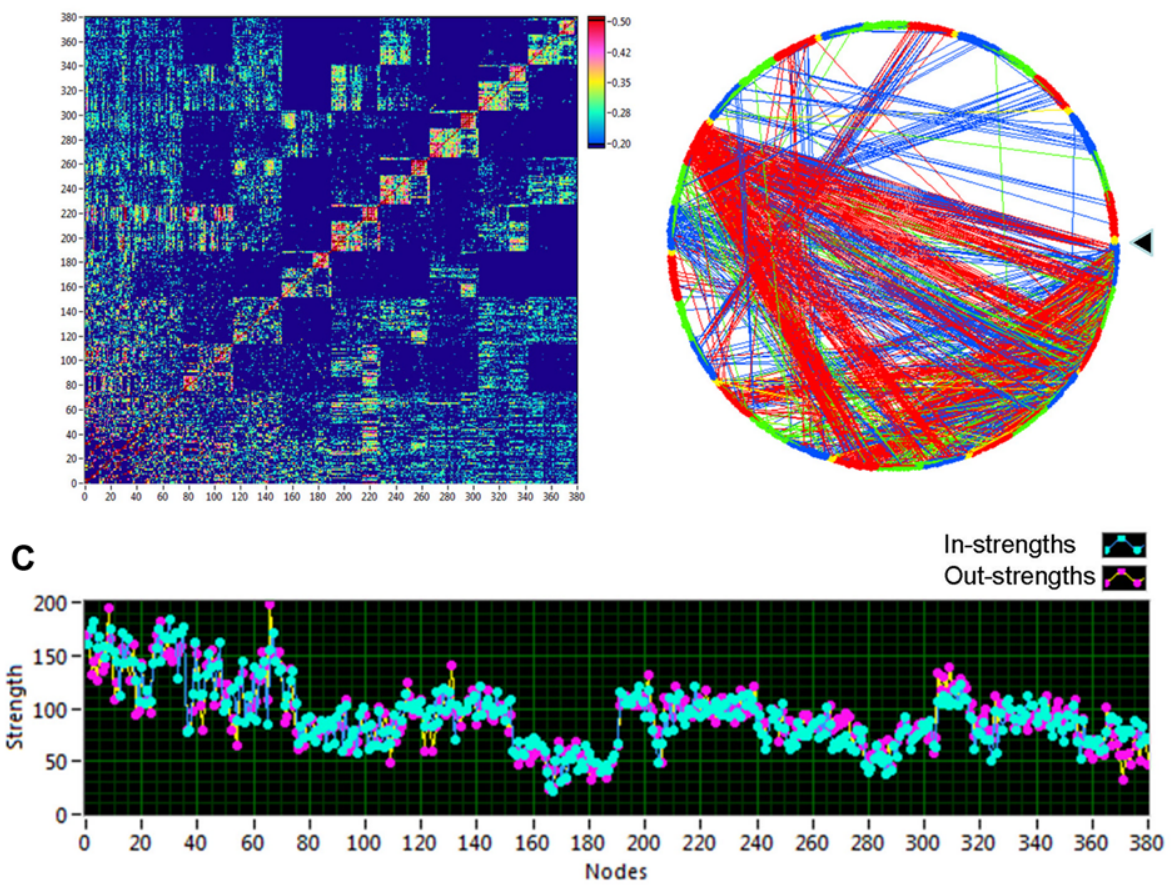

D

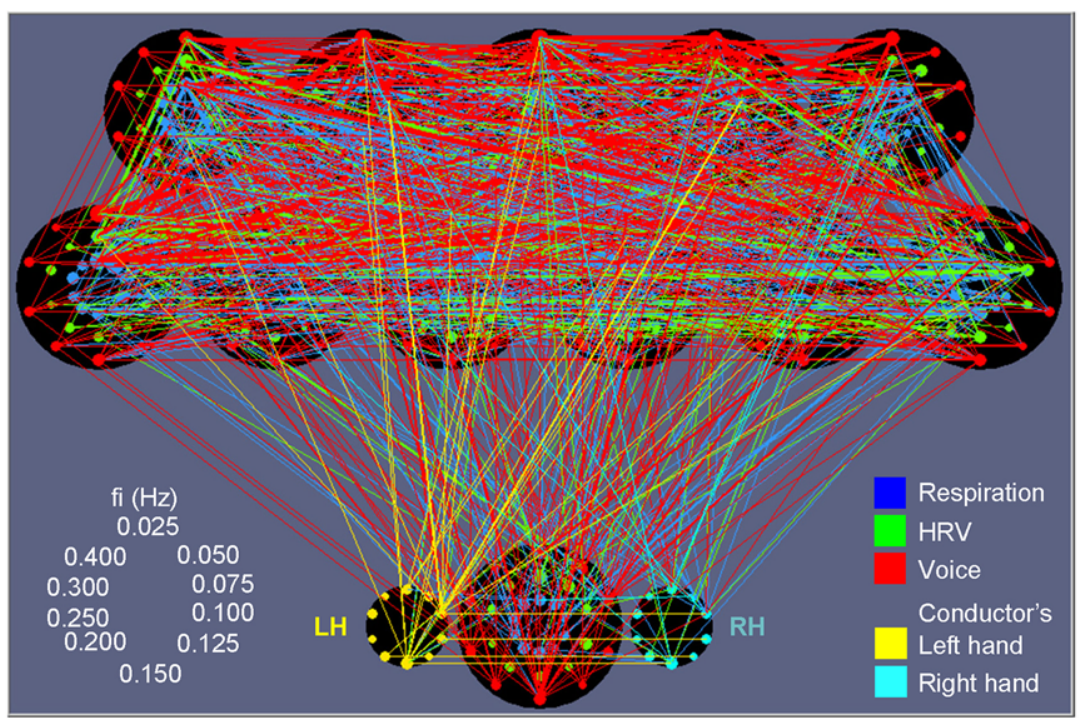

Figure 2. Common network representation. (A) Phase synchronization (ICI) matrix with 380 nodes (subsystems $x$ frequency): each frequency contains 38 nodes (12 respiration $+12 \mathrm{HRV}+12$ voice +2 hand movement nodes). (B) Common network with the strongest links $(I C I>0.4)$ represented as a circle; the order of nodes is the same as in $(\mathrm{A})$ ordered clockwise beginning from the arrow on the right. The different subsystems are color-coded: respiration, blue; HRV, green; voice, red; conductor's hand movement, yellow. (C) In- and out-strengths of the 380 nodes ordered as previously described. (D) Choir network with 11 choir members (top) and the conductor (bottom); each choir member contains 30 nodes for three subsystems (coded by color) and 10 frequencies (ordered clockwise beginning from the top; see schema on the left). The conductor also yields 10 frequency nodes for the left and the right hand (LH and RH), correspondingly. The different subsystems are color-coded as in (B). Each node's circle size reflects that node's out-strength. The connectivity strength between the nodes is indicated by the width of the line, and the color corresponds to the outgoing link. 
the conductor's hand movements. Figure 2 displays the ICI-coupling matrix (Fig. 2A), circle network (Fig. 2B), and in/out-strengths of all 380 nodes in the common network (Fig. 2C) while singing a canon with different entries. In addition, the strongest connections between choir members and the conductor are visualized (Fig. 2D). Different subsystems are coded by color, with the conductor's left and right hand displayed separately. It can be seen that WFC $(38 \times 38$ squares along the diagonal) is strongest (Fig. 2A). However, CFC also plays a crucial role in the network. Interestingly, low-frequency oscillations (e.g., 0.025 and $0.050 \mathrm{~Hz}$ ) as well as oscillations at the frequency of $0.15 \mathrm{~Hz}$ seem to be the binding forces in the network (Fig. 2B). In- and outstrengths in the common network decrease with increasing frequency (Fig. 2C). The choir members and the conductor are strongly interconnected across the subsystems (Fig. 2D). Note also that the right hand of the conductor is strongly influenced by her left hand (unidirectional coupling). Furthermore, the connections between the conductor's left hand and the choir members are mostly outgoing (i.e., from the hand to the choir), whereas the right hand contains both in- and out-going connections. This result is in line with the fact that the conductor is left-handed. The conductor's other subsystems, especially respiration and voice production, also play a crucial role in the choral network organization.

\section{Coupling strengths}

As displayed in Table 1, representing four-way repeated measure ANOVAs for WFC and CFC strengths, all main effects (except the main effect member for CFC) were statistically significant. The within- and between-member connectivity only differed in the case of WFC, whereas CFC strengths did not differ within and between the members (Fig. 3). The main effect of condition showed different change directions for the WFC and CFC strength (Fig. 3A). As in our previous analysis, ${ }^{8}$ WFC strengths $(S)$ were greater during canon singing in unison than during canon singing in parts, at least with eyes closed, as indicated by Scheffé posthoc tests: $S_{\text {Cun }}>S_{\mathrm{Cec}}, P=0.0027$. This effect is due to the between-member connectivity (Fig. 3A). In contrast, the CFC strengths were highest when singing the canon in parts and lowest when singing the canon in unison: $S_{\text {Ceo }}>S_{\text {Cun }}, P<0.0001$;
$S_{\text {Cec }}>S_{\text {Cun }}, P=0.0012$. These differences between singing conditions were also modulated by system and frequency for CFC and by frequency for WFC (Table 1). The main effect of the system (Fig. 3B) indicated that the WFC within-member strengths were strongest for the HRV, and the WFC betweenmember strengths were strongest for the respiration subsystem; the voice subsystem showed the lowest WFC strength for both within- and betweenmember connectivity. CFC strengths were strongest for the respiration and voice subsystems, and lowest for HRV for both within- and between-member connectivity. The main effect of frequency shows that CFC strengths decreased with higher frequency, whereas WFC strengths increased with higher frequency, especially for between-member connectivity (Fig. 3C).

\section{WFC network structures}

Prior to representing the complex structure of the common network, we first describe small WFC networks, which provide some interesting insights into synchronization mechanisms emerging during choral singing. For this representation, we chose two frequency components that are, as mentioned above, binding or integrative forces in the network (i.e., 0.025 and $0.150 \mathrm{~Hz}$ ) but whose WFC subnetworks seem to be different. If the edges are regularly distributed between the nodes in the low-frequency subnetwork (i.e., $0.025 \mathrm{~Hz}$ ), the coupling between voice nodes and other nodes (e.g., respiration and HRV) is strongly reduced in the high-frequency subnetwork (i.e., $0.150 \mathrm{~Hz}$ ). As can be gathered from Figure 2, these network structures are to some extent representative for low- and high-frequency components.

The upper part of Figure 4 (A-C) displays network structures at the lowest frequency $(0.025 \mathrm{~Hz})$ when singing the canon in parts with eyes closed (Fig. 4A). We used modularity analyses to partition the WFC network into different modules that are indicated by color (Fig. 4B). Thereafter, we separately partitioned the particular subsystems (respiration, HRV, and voice) into further smaller modules (Fig. 4C). The other two conditions (i.e., singing the canon in parts with eyes open and in unison) are displayed in Supplementary Figures $S 1$ and S2 (available online only). The different parts sung in the choir are recognizable in the WFC network for respiration and voice subsystems, especially 
Table 1. Statistical analysis (ANOVA) results for WFC and CFC strengths with regard to connectivity within and between the choir members

\begin{tabular}{|c|c|c|c|c|c|c|c|}
\hline \multirow[b]{2}{*}{ Effects } & \multirow[b]{2}{*}{ DF } & \multicolumn{3}{|c|}{ WFC strength } & \multicolumn{3}{|c|}{ CFC strength } \\
\hline & & $F$ value & $P$ value & Partial $\eta^{2}$ & $F$ value & $P$ value & Partial $\eta^{2}$ \\
\hline Condition (C) & 2.22 & 7.85 & 0.004 & 0.42 & 20.55 & $<0.0001$ & 0.65 \\
\hline Member (M) & 1.11 & 1314.74 & $<0.0001$ & 0.99 & 0.51 & 0.49 & 0.04 \\
\hline System (S) & 2.22 & 172.33 & $<0.0001$ & 0.94 & 36.58 & $<0.0001$ & 0.77 \\
\hline Frequency $(\mathrm{F})$ & 9.99 & 32.95 & $<0.0001$ & 0.75 & 128.26 & $<0.0001$ & 0.92 \\
\hline $\mathrm{C} \times \mathrm{M}$ & 2.22 & 12.45 & 0.001 & 0.53 & 0.45 & 0.64 & 0.04 \\
\hline $\mathrm{C} \times \mathrm{S}$ & 4.44 & 1.68 & 0.17 & 0.13 & 3.96 & 0.014 & 0.26 \\
\hline $\mathrm{C} \times \mathrm{F}$ & 18.198 & 3.72 & 0.006 & 0.25 & 3.40 & 0.007 & 0.24 \\
\hline $\mathrm{M} \times \mathrm{S}$ & 2.22 & 77.10 & $<0.0001$ & 0.88 & 0.32 & 0.73 & 0.03 \\
\hline $\mathrm{M} \times \mathrm{F}$ & 9.99 & 11.47 & $<0.0001$ & 0.51 & 2.65 & 0.028 & 0.19 \\
\hline $\mathrm{S} \times \mathrm{F}$ & 18.198 & 17.27 & $<0.0001$ & 0.61 & 10.06 & $<0.0001$ & 0.45 \\
\hline $\mathrm{C} \times \mathrm{M} \times \mathrm{S}$ & 4.44 & 1.42 & 0.26 & 0.11 & 0.74 & 0.57 & 0.06 \\
\hline $\mathrm{C} \times \mathrm{M} \times \mathrm{F}$ & 18.198 & 3.00 & 0.018 & 0.21 & 0.78 & 0.72 & 0.07 \\
\hline $\mathrm{C} \times \mathrm{S} \times \mathrm{F}$ & 36.396 & 1.70 & 0.12 & 0.13 & 2.06 & 0.051 & 0.16 \\
\hline $\mathrm{M} \times \mathrm{S} \times \mathrm{F}$ & 18.198 & 3.59 & 0.005 & 0.25 & 1.32 & 0.25 & 0.11 \\
\hline $\mathrm{C} \times \mathrm{M} \times \mathrm{S} \times \mathrm{F}$ & 36.396 & 2.01 & 0.062 & 0.15 & 0.89 & 0.53 & 0.08 \\
\hline
\end{tabular}

WFC, within-frequency coupling; CFC, cross-frequency coupling.

when singing the canon with eyes closed, when the conductor sang along with the third entry group. In the smaller networks of separate subsystems, only the respiration subsystem allows the recognition of different parts sung in the choir. In the voice subsystem, only the first entry could be separated when singing the canon with eyes open or closed, whereas the second and the third entries were in the same module. Likewise, in the HRV subsystem, at least during canon singing with eyes open, only the third entry could be separated, whereas the first and second entries were in the same module (except for participant 5 , who built a separate module). Most interestingly, in the WFC network (especially when singing the canon with eyes closed), the voice production nodes of the choir members singing the first entry shared the same module with the respiration nodes of the choir members singing the second entry (Fig. 4B). Accordingly, the voice production nodes of the choir members singing the second entry shared the same module with the respiration nodes of the choir members singing the third entry, etc. In other words, the strongest connections between voice and respiration systems were shifted in time: respiration of the choir members singing the entry was connected to and apparently oriented on the voices of the choir members singing the previous entry. The inspection of community structures of the choir singing in unison (Fig. S2B, available online only) showed only two modules that mainly separated the respiration and voice production subsystems. HRV nodes were distributed across both modules. More interestingly, the coupling between respiration and voice subsystems was mostly unidirectional, with intermodular connections going from respiration to voice nodes.

Next, we inspected WFC networks at the frequency of interest of $0.150 \mathrm{~Hz}$. WFC networks at this frequency presented in the bottom part of Figure 4 (D-F) for singing the canon with eyes closed showed that (1) connections going from the voice subsystem to the respiration and HRV subsystems were practically absent (interestingly, there were some connections coming from respiration and HRV to the voice subsystem); and (2) choir members within the voice subsystem (as well as within the other subsystems) were strongly interconnected. A modularity analysis separated the voice subsystem from the other subsystems, indicating strong connectivity within the two modules and only few intermodular connections coming from the other subsystems to the voice subsystem. The other two conditions (i.e., singing the canon in parts with eyes open or in unison), that are displayed in the bottom part of Supplementary Figures S1 and S2 (available online only), showed similar results, with 
A
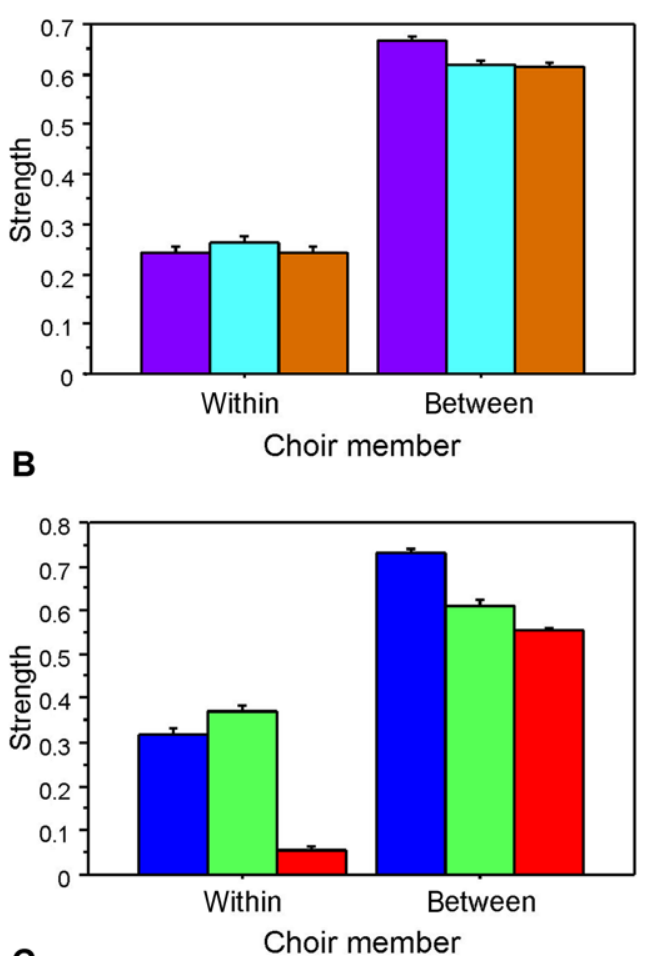

C

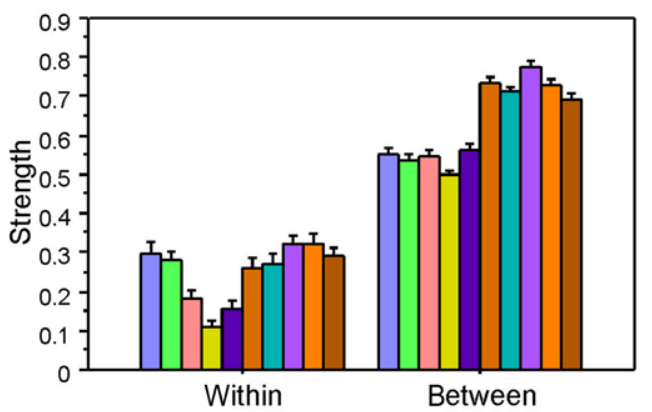

Choir member
CFC
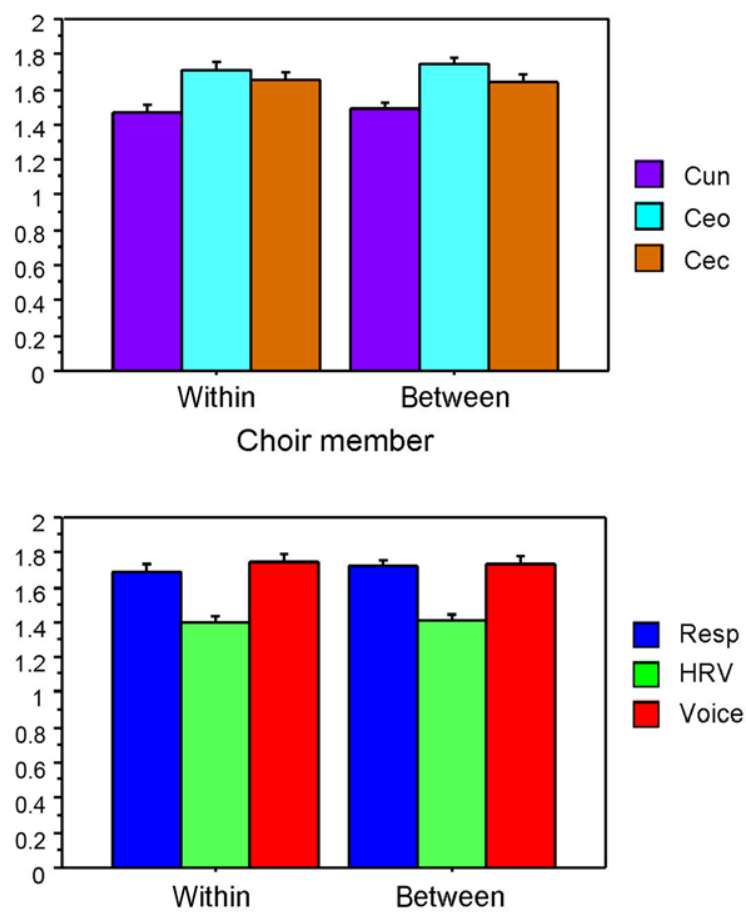

Choir member

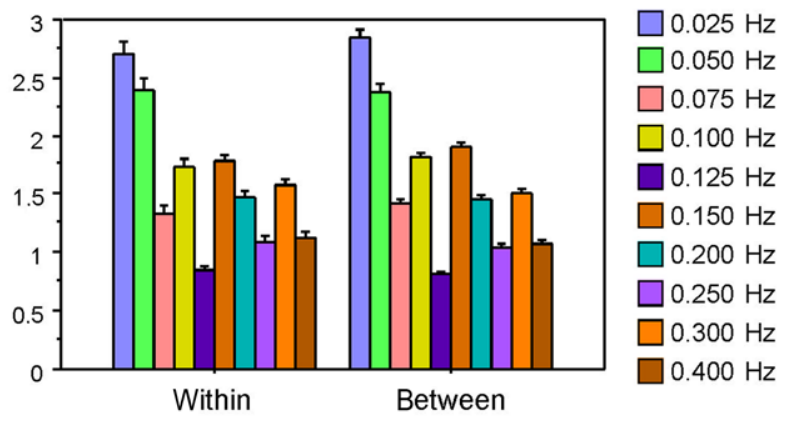

Choir member

Figure 3. Out-strengths for the WFC (left) and the CFC connections (right). (A) Out-strengths for the within- and betweenmember connectivity under the three canon conditions: $\mathrm{C}_{\mathrm{un}}$ - singing canon in unison, $\mathrm{C}_{\mathrm{eo}}$ - singing canon with eyes open, and $\mathrm{C}_{\mathrm{ec}}$ - singing canon with eyes closed. (B) Out-strengths for the within- and between-member connectivity and the three subsystems: respiration (Resp), heart rate variability (HRV), and voice production (Voice). (C) Out-strengths for the within- and betweenmember connectivity and the 10 different frequencies of interest. Please note that on the right, the displayed CFC connections are averaged across all member pairs.

the difference that the voice subsystem is practically disconnected from the other subsystems (there are only few incoming connections) in the unison condition. A modularity analysis of each particular subsystem was not able to partition it into different modules $(M=0)$ but there was a conductor effect in the respiration subsystem with the connections that were predominantly directed from the conductor to the choir members, especially during canon singing with eyes open. The voice subsystem also showed strong, mostly bidirectional connections to and from the conductor. This indicates that changes 


$$
\mathrm{FOI}=0.025 \mathrm{~Hz}
$$

A

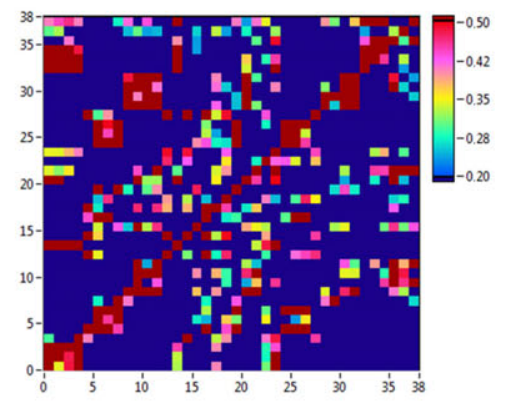

B Modularity structure of the network

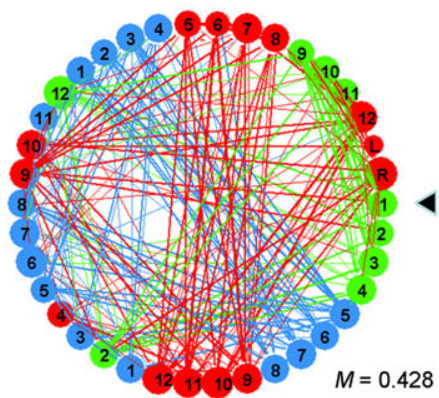

C

Modularity structures of different subsystems within the network

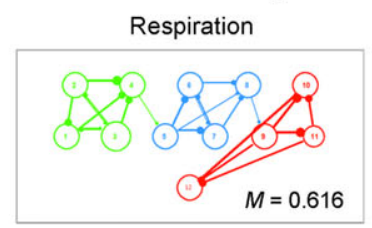

HRV

Voice
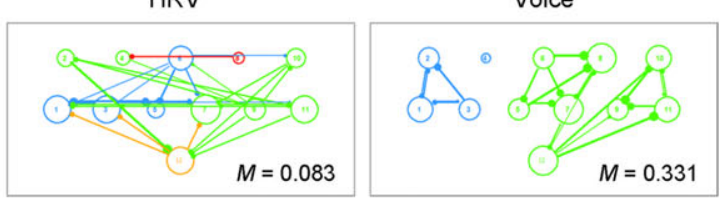

$$
\mathrm{FOI}=0.150 \mathrm{~Hz}
$$

D

WFC network

E

Modularity structure of the network
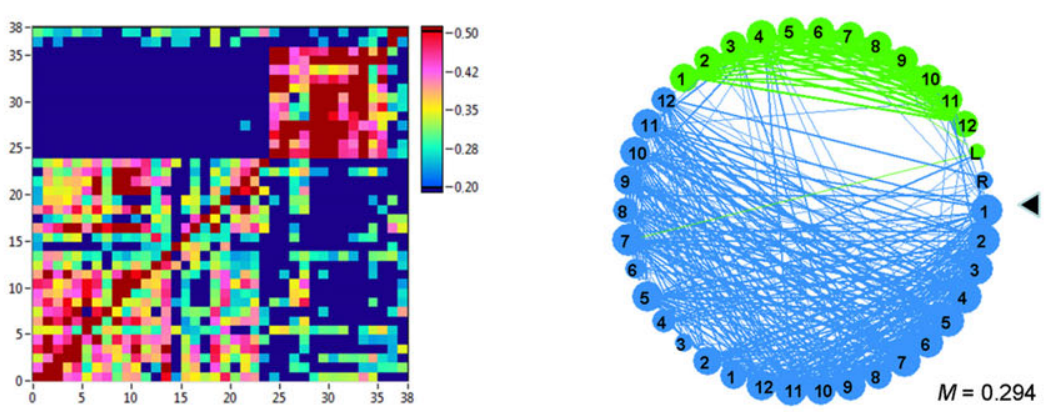

$\mathbf{F}$

Connectivity strengths between the choir members and the conductor

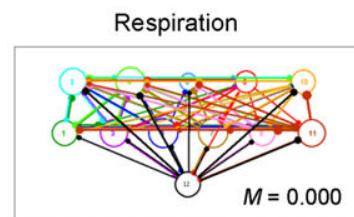
HRV
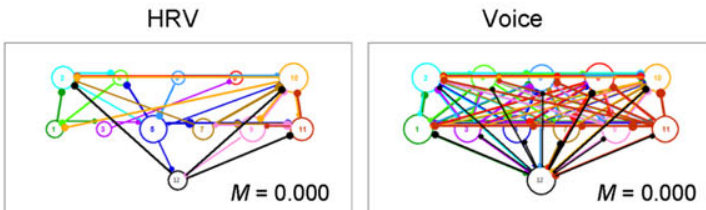

Figure 4. WFC networks and corresponding modularity structures for canon singing with eyes closed. (A) WFC network, (B) modularity structure of the network, and (C) modularity structures of the different subsystems within the network at FOI $=$ $0.025 \mathrm{~Hz}$. (D) WFC network, (E) modularity structure of the network, and (F) modularity structures of the different subsystems within the network at FOI $=0.150 \mathrm{~Hz}$. Network matrix in (A) and (D) with 38 nodes represents the choir members' and the conductor's subsystems (12 respiration $+12 \mathrm{HRV}+12$ voice +2 hand movement nodes). Nodes in (B) and (E) are ordered clockwise beginning from the arrow on the right, with the node numbering from 1 to 11 corresponding to the choir members and 12 indicating the conductor. The order of subsystems corresponds to that in (A) and (D): respiration, HRV, voice, and hand movement nodes. The colors in (B), (C), and (E) represent the different modules, whereas in (F) they correspond to different choir members (the modularity in this case equals zero, meaning that there are no modularity structures). FOI, frequency of interest; HRV, heart rate variability. 
in respiration patterns occurred earlier in the conductor than in the choir members and were transmitted from the conductor to the choir. This pattern is of course in accordance with the conductor's functional role in a choir. The inspection of community structures of singing in unison (Fig. S2F, available online only) revealed very similar results.

\section{WFC-CFC network structures}

Modularity analyses partition the common (i.e., WFC-CFC) network into four different modules for all three canon conditions. Figure 5 represents the modularity structure of the choir with network connections within each choir member (Fig. 5A) and between them (Fig. 5B) when singing the canon with different entries with eyes open (corresponding network structures for the other two canon conditions are presented in Figs. S3 and S4, available online only). The different modules are coded by color, the circle size of different sensors displays the node strength, and the line width corresponds to the connectivity or edge strength. It can be seen that connectivity between the choir members was much stronger than between the different subsystems within each of the choir members (note that connections within the choir members are displayed at a much lower threshold than those between them). Furthermore, all choir members contained all four modules partitioned in the network. The modularity structure of each choir member resembled the others' - with some variants and invariants in modular organization. The low-frequency nodes of all subsystems varied in their subdivision into the modules, whereas the high-frequency nodes were mostly invariant across choir members.

To investigate the role of different subsystems and frequencies in the modular organization of the choir network, we calculated the within-module degree $\left(Z_{i}\right)$, indicating how well node $i$ is connected to other nodes within the module of interest $\left(m_{i}\right)$, and the participation coefficient $\left(P_{i}\right)$, indicating how well-distributed the links of the node $i$ are among the other modules. ${ }^{29}$ Together, the within-module degree and the participation coefficient form the socalled $Z-P$ parameter space and define the role of the nodes in modular organization of the network. The within-module degree distinguishes between hubs and nonhubs, while the participation coefficient discriminates between provincial and connector nodes. Figure 6 displays the $Z-P$ parameter space of the choir network across the three canon conditions (Fig. 6A-C), where different nodes are coded by color for different subsystems (left) and frequency (right) components. One can see that the respiration subsystem and low-frequency components play an important role regarding both hub (especially respiration nodes) and connector (especially lowfrequency nodes) properties. The three canon conditions were significantly different only in the case of $P_{i}$ values, with the highest $P_{i}$ value obtained during canon singing with different entries and the lowest reached during canon singing in unison (Scheffé post-hoc tests: $P i_{\text {Ceo }}>P i_{\text {Cec }}, P<0.0001 ; P i_{\text {Cec }}>$ $\left.P i_{\text {Cun }}, P<0.0001\right)$. Statistical analyses also revealed significant differences between the three subsystems for both $Z_{i}$ and $P_{i}$ values (see Table 2 for details): The $Z_{i}$ value was highest for the respiration subsystem and lowest for the voice subsystem, as indicated by Scheffé post-hoc tests: $Z i_{\text {resp }}>Z i_{\mathrm{HRV}}, P<0.0001$; $Z i_{\text {resp }}>Z i_{\text {voice, }} P<0.0001$, whereas the $P_{i}$ value was highest for the voice subsystem and lowest for HRV: $P i_{\text {voice }}>P i_{\text {resp }}, P<0.0001 ; P i_{\text {resp }}>P i_{\mathrm{HRV}}$, $P<0.0001$. Thus, the respiration subsystem showing more hub properties (high $Z_{i}$ values) provides for connectivity within and between the modules, whereas the voice subsystem is responsible above all for intermodular connectivity. Generally, $P_{i}$ values for low-frequency components were higher than for high-frequency components, except for nodes of 0.150 and $0.300 \mathrm{~Hz}$. In contrast, $Z_{i}$ values were highest for high-frequency components, especially for frequency components of $0.150,0.200$, and $0.250 \mathrm{~Hz}$ (see Fig. 6 for details). Significant interactions, condition $\times$ system, condition $\times$ frequency, condition $\times$ system $\times$ frequency, for both $Z_{i}$ and $P_{i}$ values (see Table 2 for details), indicate that $Z_{i}$ values, at least for the respiration subsystem, and $P_{i}$ values for all subsystems were higher during canon singing in parts than when singing the canon in unison. This means that the number of hub and connector nodes increased when the canon was sung in parts as compared to being sung in unison (Fig. 6).

\section{Discussion}

We examined the oscillatory network topography of choir singing as indexed by cardiac, respiratory, vocalizing, and gestural activity to investigate two main questions. First, is there evidence to suggest that the respiratory and cardiac subsystems of the singers synchronize during singing and couple to 
A

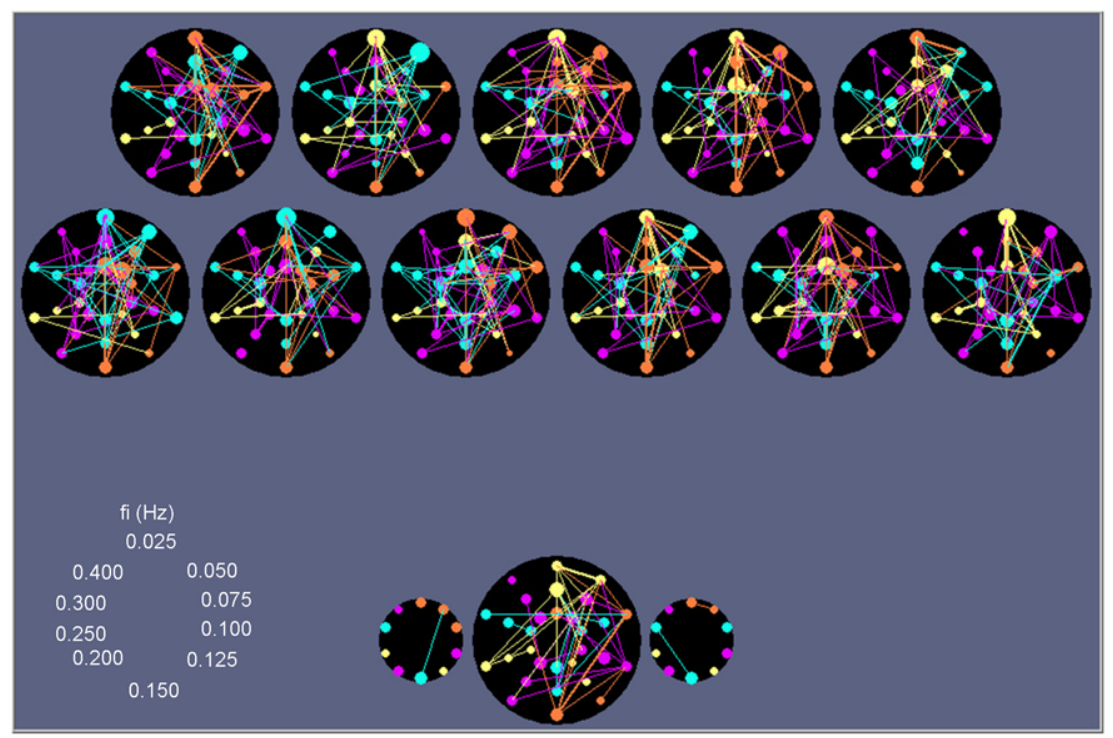

B

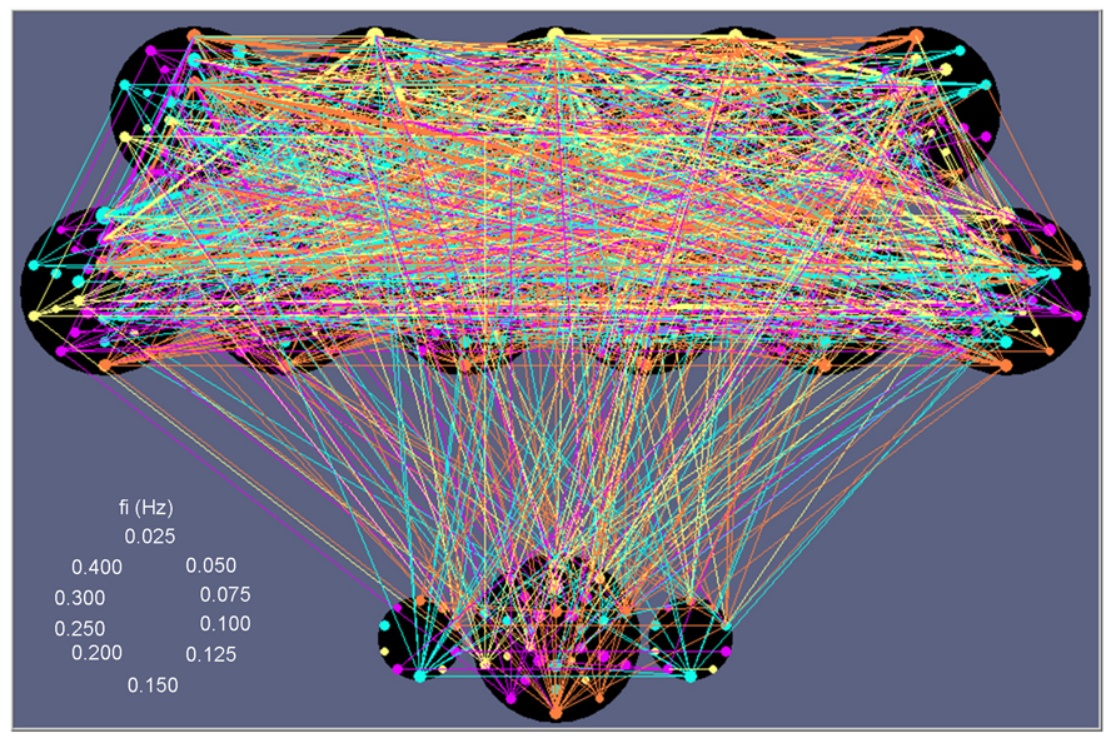

Figure 5. Modularity structure during canon singing with eyes open, showing the strongest connections within and between the choir members and the conductor. (A) Network connectivity within the choir members and the conductor. (B) Network connectivity between the choir members and the conductor. Different modules are color-coded. Each node's circle size reflects its out-strength. The connectivity strength between the nodes is given by line width, and is color-coded corresponding to the outgoing link. Note that the connectivity threshold is different: 0.4 and 0.5 in (A) and (B), respectively.

the vocalizing patterns of the singers, and to the hand movements of the choir's conductor? Second, and more specifically, do the oscillatory networks within individual singers vary as a function of choir context, in line with the hypothesis that the choir functions as a superordinate system? Our analyses provide affirmative answers to both questions.

We found that all investigated subsystems were synchronized with each other both within and between different frequencies as well as within 
A

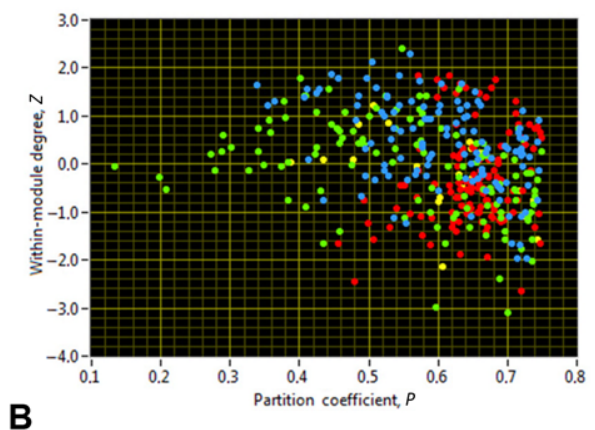

B
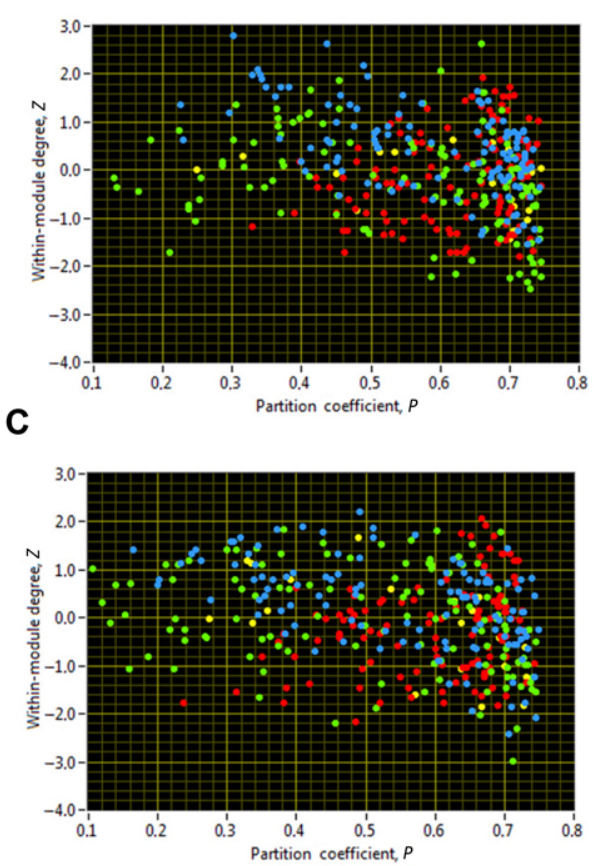

Frequency components
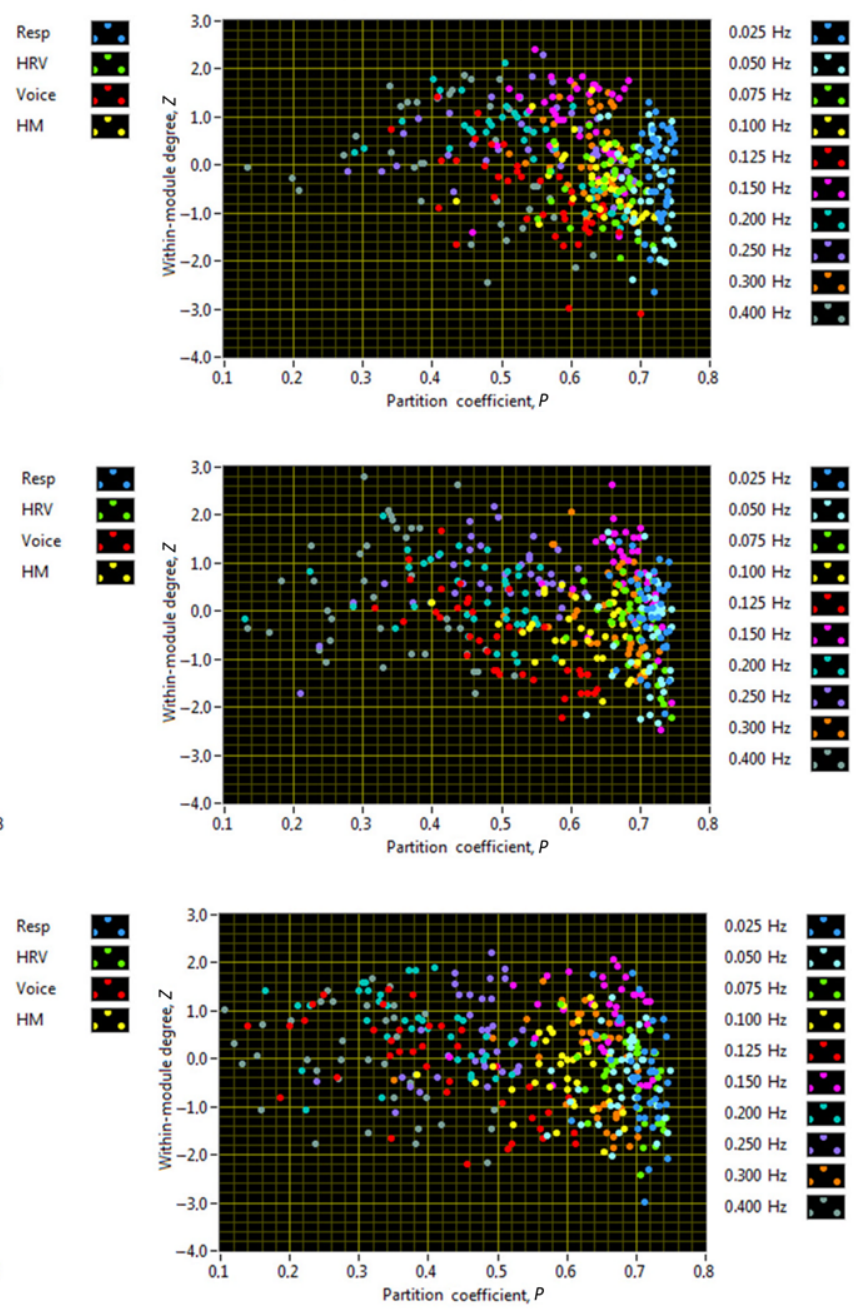

Figure 6. Distribution of nodes in a $Z-P$ parameter space for the three different canon conditions indicated for the different subsystems and frequency components. (A) Canon singing with eyes open, (B) canon singing with eyes closed, and (C) canon singing in unison. The colors correspond to the different subsystems (left) and frequency components (right). The within-module degree $Z_{i}$ indicates nodes' connectivity within the module, and the participation coefficient $P_{i}$ indicates nodal connections across different modules. Resp, respiration; HRV, heart rate variability; HM, hand movement.

and between individuals. Separate analyses of WFC and CFC strengths with regard to within- and between-member connectivity revealed stronger between-member connectivity relative to withinmember connectivity only in the case of WFC, whereas CFC strengths were similar for within- and between-member connectivity. Most importantly, CFC strengths with regard to both the within- and between-member connectivity were highest when singing the canon in parts and lowest when singing the canon in unison, while WFC was strongest when singing in unison and only for between-member connectivity. This result, indicating that all the subsystems synchronize more strongly within the same frequency when the same entry of the canon was sung by the whole choir, is not surprising and is in accordance with our earlier analyses regarding respiration and HRV. ${ }^{8}$ The former result suggests that choir members singing the canon in parts increasingly synchronized their actions or involved 
Table 2. Statistical analysis (ANOVA) results for within-module degree $\left(Z_{i}\right)$ and participation coefficient $\left(P_{i}\right)$

\begin{tabular}{|c|c|c|c|c|c|c|c|}
\hline \multirow[b]{2}{*}{ Effects } & \multirow[b]{2}{*}{ DF } & \multicolumn{3}{|c|}{$Z_{i}$} & \multicolumn{3}{|c|}{$P_{i}$} \\
\hline & & $F$ value & $P$ value & Partial $\eta^{2}$ & $F$ value & $P$ value & Partial $\eta^{2}$ \\
\hline Condition (C) & 2.22 & 0.001 & 0.998 & 0.00 & 156.38 & $<0.0001$ & 0.93 \\
\hline System (S) & 2.22 & 36.11 & $<0.0001$ & 0.77 & 142.56 & $<0.0001$ & 0.93 \\
\hline Frequency (F) & 9.99 & 27.90 & $<0.0001$ & 0.72 & 345.49 & $<0.0001$ & 0.97 \\
\hline $\mathrm{C} \times \mathrm{S}$ & 4.44 & 5.56 & 0.0030 & 0.34 & 3.59 & 0.020 & 0.25 \\
\hline $\mathrm{C} \times \mathrm{F}$ & 18.198 & 2.22 & 0.038 & 0.17 & 23.71 & $<0.0001$ & 0.68 \\
\hline $\mathrm{S} \times \mathrm{F}$ & 18.198 & 20.71 & $<0.0001$ & 0.65 & 19.16 & $<0.0001$ & 0.64 \\
\hline $\mathrm{C} \times \mathrm{S} \times \mathrm{F}$ & 36.396 & 1.69 & 0.11 & 0.13 & 2.82 & 0.011 & 0.20 \\
\hline
\end{tabular}

subsystems at different frequencies, that is, crossfrequency interaction plays a crucial role in this case. With regard to different subsystems, the CFC strengths were highest for the respiration and voice subsystem, and lowest for HRV, while the WFC strengths were highest for HRV within members and for respiration between members but lowest for the voice subsystem, especially for within-member connectivity. Thus, the voice subsystem interacts with other subsystems, especially within individual singers, through cross-frequency connections.

Thus, CFC supports canon singing in parts both on the individual and on the group level, whereas WFC is important for consolidation of the choir when all of its members are singing the same canon part in unison. In accordance with these coupling changes, we also observed corresponding changes in network topology when investigating modularity structures and $Z-P$ parameter space underlying these structures. Specifically, the number of hub and connector nodes in the network increased when the canon was sung in parts as compared to singing in unison, while the singing conditions did not differ in the number of modules in the network. Hubs indicated by high within-module degree are characterized by strong connectivity within the modules, whereas connectors indicated by a high participation coefficient are responsible for strong connectivity between different modules. It is clear that singing a canon in parts represents a more complex coordination action and therefore requires more hub and/or connector nodes. In line with increased CFC, the increased number of hub and connector nodes when singing a canon in parts is clearly due to CFC connections. Modules detected by modularity analyses were heterogeneous, consisting of different subsystems and including all choir members.
Notwithstanding the important role of CFC in choir singing, there were interesting effects with regard to the interaction between different subsystems when considering WFC at particular frequencies. The modularity analyses of the WFC networks at low frequencies (e.g., $0.025 \mathrm{~Hz}$ ) revealed intriguing interactions between respiration and voice subsystems connecting choir members singing different canon entries. Specifically, the voice production nodes of the choir members singing the first entry shared the same module with the respiration nodes of the choir members singing the second entry. Accordingly, the voice production nodes of the choir members singing the second entry shared the same module with the respiration nodes of the choir members singing the third entry, etc. In other words, the strongest connections between voice and respiration subsystems are shifted in time: the voices of the choir members singing the previous entry are connected to the respiration of the choir members singing the next entry. Apparently, there is a dynamic interaction between the voice and respiration subsystems: at the time when one group of choir members sing an entry, the next group of choir members inhale to sing the following entry. As to be expected, we did not find such a WFC network modularity structure during the singing of the canon in unison; in this case, the modularity structure was more amorphous $(M=0.230)$ and contained only two modules separating respiration and voice subsystems, while the HRV nodes were randomly distributed across these two modules. This cross-talking between respiration and voice subsystems, which is present only when the canon was sung in parts, represents a further mechanism on the level of small WFC networks that contributes to choir performance. 
In the light of this interaction, a further interesting effect was found for WFC at higher frequencies (i.e., $0.100 \mathrm{~Hz}$ and higher), where connections between voice and other subsystems (out-strengths) were reduced or even absent, although the synchronization between choir members within the voice subsystem (and also within the other subsystems) was intact and relatively strong at these frequencies. Modularity analysis showed that the voice subsystem was separate from other subsystems, with intermodular connections mostly going from other subsystems to the voice subsystem. This means that the voice subsystem is driven by the other subsystems at these frequencies. Interestingly, the absence or strong attenuation of WFC between the voice subsystem and other subsystems is compensated by CFC to lower frequencies (i.e., $0.025-0.075 \mathrm{~Hz}$ ). In other words, voice production at a high frequency communicates with other subsystems oscillating at lower frequencies. Characteristic for higher frequencies is the so-called conductor effect within the respiration subsystem that we also described in our previous paper. ${ }^{8}$ The voice subsystem also showed strong conductor connections but these were mostly bidirectional. This is not surprising because the conductor sang along with other choir members and was strongly synchronized with them. Changes in respiration patterns occurred earlier in the conductor than in the choir members, and were thus transmitted from the conductor to the choir. Another interesting directionality effect is that of the conductor's hand movements. The left hand of the conductor showed unidirectional connections not only to practically all choir members but also to her right hand. There were also unidirectional connections between the right hand and the choir members but in contrast to the left hand, the right-hand nodes contained both in- and out-going connections. This result is of particular interest because the conductor was left-handed and her left hand was the leading one with regard to conducting. These directionality patterns are in accordance with the conductor's functional role in a choir and emphasize his/her group leader or group facilitator characteristics.

As mentioned, modularity analyses showed that the common choir network could be subdivided into four different modules for all three canon conditions. It is remarkable that all modules contain all four subsystems investigated in the study and always include low-frequency oscillations (i.e., 0.025 and $0.050 \mathrm{~Hz}$ ), which probably fulfill an integrative function in the network. As shown earlier, the next integrating frequency is the $0.150 \mathrm{~Hz}$ oscillation, which revealed strong local (WFC) and global (CFC) connectivity strengths. Our interbrain synchronization study, ${ }^{12}$ in which we used CFC to construct hyperbrain networks, showed that alpha brain oscillations fulfill such an integrating function in the hyperbrain networks occurring when romantic couples kiss. Thus, we can assume that complex networks (biological or social) constructed using CFC algorithms and allowing investigation of a complex interplay between different frequencies reveal frequencies playing a leading or integrative role in the network. In the choir network, there were several frequencies (e.g., $0.025,0.050$, or $0.150 \mathrm{~Hz}$ ) that could play this integrative role, thus constituting the choir as a coherent entity or superorgan. This integration can occur at different system and network levels. Furthermore, some frequencies play a specific role in the network. For example, high frequencies, as mentioned above, support the causal effect of the conductor on the choir members, especially from her respiration or hand movement subsystem, representing continuous interventions of the conductor to stabilize the choir's singing pace.

The investigation of the within-module degree and participation coefficient for the different nodes showed that the former was highest for the respiration subsystem and lowest for the voice subsystem, whereas the latter was highest for the voice subsystem and lowest for HRV. Presumably, the respiration subsystem (or at least some of the respiration nodes) predominantly assumes the role of network hubs, which are responsible for links within the modules, whereas the voice subsystem mainly assumes the role of network connectors, which support the connectivity between different modules. Interestingly, hubs are more likely to oscillate at the middle or higher frequencies (e.g., $0.150,0.200$, or $0.250 \mathrm{~Hz}$ ), whereas connectors are more likely to use the low frequencies $(0.025,0.050$, or $0.075 \mathrm{~Hz})$. The oscillation frequency of $0.150 \mathrm{~Hz}$ revealed relatively high numbers of both hub and connector nodes. As mentioned above, these also exhibit a strong local (WFC) and global (CFC) connectivity strengths. This only confirms our former suggestion that the $0.150-\mathrm{Hz}$ oscillation plays a crucial role in network integration. Low-frequency nodes integrate the network mostly as connectors, although there are some nodes 
that also possess both hub and connector properties, that is, binding the nodes within and between the modules. Thus, this analysis of choral singing shows the complex interplay of different subsystems and frequency components that also interact in different ways in different canon conditions.

Caveats aside, the present findings are important as they support the idea that a choir as a group of interacting individuals exhibits collective behavior and thus functions as a superorganism or a superordinate system. ${ }^{18,30,31}$ The network dynamics of each individual singer are likely to be influenced by a complex coordination or the function of the choir as a whole. At the same time, each singer contributing to the choir tends to bring under control the superordinate system itself. It has been shown that coupling or integrative dynamics of the superordinate system at lower levels of individuality (e.g., within-member CFC) display similar synchronization patterns for interaction within the system itself (e.g., between-member CFC). Such symmetries at individual and system levels and a certain balance between bottom-up and top-down influences in the system seem to be crucial for social interaction and collective behavior in animals and humans. ${ }^{18,30}$

The present study has limitations and leaves room for questions to be addressed in future research. First, the sample size of our study was small. Further studies with larger samples would provide additional and more reliable information about coupling mechanisms during singing and would enhance the generalizability of the findings. Second, we examined a group of participants who were used to singing together regularly (albeit in an amateur choir), and our findings may not extend to spontaneous group singing. Moreover, we do not know if it is uniquely singing that leads to the effects found in the study or whether other musical or nonmusical coordinated breathing activities could achieve similar results. Third, our measurements were restricted to ANS responses. Using electroencephalographic or near-infrared spectroscopy recordings would provide further important information about brain dynamics and enhance the interpretability of results.

Our results extend previous work on the reach of network interactions during choir singing and highlight the way in which CFC integrates different subsystems during temporally coordinated forms of social interaction. These findings are in line with studies investigating neural markers of dynamic real-world group interactions, ${ }^{32}$ rhythmic interpersonal coordination, ${ }^{33}$ and sensorimotor coupling in music. ${ }^{34,35}$ Our methods offer a toolkit for the study of interpersonal action coordination across a wide range of coordinated social activities.

\section{Acknowledgments}

We thank the conductor of the Max Planck Institute for Human Development choir, Juliane Oppelt, and the choir members for their participation in the study. The technical assistance of Anja Lender, Bibiana Klempova, Charlotte Küpper, Milena Koring, and Sebastian Schröder during data acquisition is greatly appreciated. We also thank Berndt Wischnewski for software assistance.

\section{Supporting information}

Additional supporting information may be found in the online version of this article.

Figure S1. WFC networks and corresponding modularity structures for canon singing with eyes open.

Figure S2. WFC networks and corresponding modularity structures for canon singing in unison.

Figure S3. Modularity structure during canon singing with eyes closed showing the strongest connections within and between the choir members and the conductor.

Figure S4. Modularity structure during canon singing in unison showing the strongest connections within and between the choir members and the conductor.

\section{Competing interests}

The authors declare no competing interests.

\section{References}

1. Clift, S. \& G. Hancox. 2010. The significance of choral singing for sustaining psychological wellbeing: findings from a survey of choristers in England, Australia and Germany. Music Perform. Res. 3: 79-96.

2. Grape, C., M. Sandgren, L.-O. Hansson, et al. 2003. Does singing promote well-being? An empirical study of professional and amateur singers during a singing lesson. Integr. Physiol. Behav. Sci. 38: 65-74.

3. Livesey, L., I. Morrison, S. Clift, et al. 2012. Benefits of choral singing for social and mental wellbeing: qualitative findings from a cross-national survey of choir members. J. Public Ment. Health 11: 10-26. 
4. Stewart, N.A.J. \& A.J. Lonsdale. 2016. It's better together: the psychological benefits of singing in a choir. Psychol. Music 44: $1240-1254$.

5. Bailey, B.A. \& J.W. Davidson. 2005. Effects of group singing and performance for marginalized and middle-class singers. Psychol. Music 33: 269-303.

6. Clift, S., G. Hancox, I. Morrison, et al. 2010. Choral singing and psychological wellbeing: quantitative and qualitative findings from English choirs in a cross-national survey. $J$. Appl. Arts Heal. 1: 19-34.

7. Silber, L. 2005. Bars behind bars: the impact of a women's prison choir on social harmony. Music Educ. Res. 7: 251-271.

8. Müller, V. \& U. Lindenberger. 2011. Cardiac and respiratory patterns synchronize between persons during choir singing. PLoS One 6: e24893.

9. Bruns, A. \& R. Eckhorn. 2004. Task-related coupling from high- to low-frequency signals among visual cortical areas in human subdural recordings. Int. J. Psychophysiol. 51:97-116.

10. Cohen, M.X. 2008. Assessing transient cross-frequency coupling in EEG data. J. Neurosci. Methods 168: 494-499.

11. Jirsa, V. \& V. Müller. 2013. Cross-frequency coupling in real and virtual brain networks. Front. Comput. Neurosci. 7: 78.

12. Müller, V. \& U. Lindenberger. 2014. Hyper-brain networks support romantic kissing in humans. PLoS One 9: e112080.

13. Witte, H., P. Putsche, C. Hemmelmann, et al. 2008. Analysis and modeling of time-variant amplitude-frequency couplings of and between oscillations of EEG bursts. Biol. Cybern. 99: 139-157.

14. Axmacher, N., M.M. Henseler, O. Jensen, et al. 2010. Crossfrequency coupling supports multi-item working memory in the human hippocampus. Proc. Natl. Acad. Sci. USA 107: 3228-3233.

15. Canolty, R.T., E. Edwards, S.S. Dalal, et al. 2006. High gamma power is phase-locked to theta oscillations in human neocortex. Science 313: 1626-1628.

16. Tort, A.B.L., R.W. Komorowski, J.R. Manns, et al. 2009. Theta-gamma coupling increases during the learning of item-context associations. Proc. Natl. Acad. Sci. USA 106: 20942-20947.

17. Tort, A.B.L., M.A. Kramer, C. Thorn, et al. 2008. Dynamic cross-frequency couplings of local field potential oscillations in rat striatum and hippocampus during performance of a T-maze task. Proc. Natl. Acad. Sci. USA 105: 20517-20522.

18. Noble, D. 2012. A theory of biological relativity: no privileged level of causation. Interface Focus 2: 55-64.

19. Konvalinka, I., D. Xygalatas, J. Bulbulia, et al. 2011. Synchronized arousal between performers and related spectators in a fire-walking ritual. Proc. Natl. Acad. Sci. USA 108: 85148519 .
20. Censi, F., G. Calcagnini, S. Lino, et al. 2000. Transient phase locking patterns among respiration, heart rate and blood pressure during cardiorespiratory synchronisation in humans. Med. Biol. Eng. Comput. 38: 416-426.

21. Tzeng, Y.C., P.D. Larsen \& D.C. Galletly. 2003. Cardioventilatory coupling in resting human subjects. Exp. Physiol. 88: 775-782.

22. Malik, M., J.T. Bigger, J.A. Camm, et al. 1996. Heart rate variability: standards of measurement, physiological interpretation, and clinical use. Eur. Heart J. 17: 354-381.

23. Schäfer, C., M.G. Rosenblum, J. Kurths, et al. 1998. Heartbeat synchronized with ventilation. Nature 392: 239-240.

24. Vickhoff, B., H. Malmgren, R. Åström, et al. 2013. Music structure determines heart rate variability of singers. Front. Psychol. 4: 334.

25. Müller, V., D. Perdikis, T. Von Oertzen, et al. 2016. Structure and topology dynamics of hyper-frequency networks during rest and auditory oddball performance. Front. Comput. Neurosci. 10: 108.

26. Rubinov, M. \& O. Sporns. 2010. Complex network measures of brain connectivity: uses and interpretations. Neuroimage 52: 1059-1069.

27. Leicht, E. \& M. Newman. 2008. Community structure in directed networks. Phys. Rev. Lett. 100: 118703.

28. Bassett, D.S., D.L. Greenfield, A. Meyer-Lindenberg, et al. 2010. Efficient physical embedding of topologically complex information processing networks in brains and computer circuits. PLoS Comput. Biol. 6: e1000748.

29. Guimerà, R. \& L.A.N. Amaral. 2005. Functional cartography of complex metabolic networks. Nature 433: 895-900.

30. Emerson, A.E. 1939. Social coordination and the superorganism. Am. Midl. Nat. 21: 182-209.

31. Trianni, V., E. Tuci, K.M. Passino, et al. 2011. Swarm cognition: an interdisciplinary approach to the study of selforganising biological collectives. Swarm Intell. 5: 3-18.

32. Dikker, S., L. Wan, I. Davidesco, et al. 2017. Brain-to-brain synchrony tracks real-world dynamic group interactions in the classroom. Curr. Biol. 27: 1375-1380.

33. Keller, P.E., G. Novembre \& M.J. Hove. 2014. Rhythm in joint action: psychological and neurophysiological mechanisms for real-time interpersonal coordination. Philos. Trans. R. Soc. B Biol. Sci. 369. https://doi.org/10.1098/rstb.2013.0394.

34. Fairhurst, M.T., P. Janata \& P.E. Keller. 2017. Being and feeling in sync with an adaptive virtual partner: brain mechanisms underlying dynamic cooperativity. Cereb. Cortex 23: 2592-2600.

35. Janata, P., S.T. Tomic \& J.M. Haberman. 2012. Sensorimotor coupling in music and the psychology of the groove. J. Exp. Psychol. Gen. 141: 54-75. 